\title{
PROPERTIES OF THE TRIANGULAR LATTICE GAS WITH REPULSIVE INTERACTIONS
}

\author{
M. SCHICK and J. S. WALKER \\ Department of Physics, University of Washington, \\ Seattle, Washington 98195 , U.S.A. \\ and

\section{WORTIS} \\ Department of Physics, University of Illinois, \\ Urbana, Illinois 61801, U.S.A.
}

\begin{abstract}
Résumé. - Le gaz à réseau triangulaire à interactions entre plus proches voisins est utilisé comme modèle pour quelques systèmes adsorbés. Nous avons appliqué à ce modèle les méthodes du groupe renormalisation et obtenu son diagramme de phase que nous avons comparé à celui de $\mathrm{He}^{4}$ adsorbé sur Grafoil. Les valeurs calculées pour la chaleur spécifique sans aucun paramètre ajustable, sont en remarquable accord avec l'expérience au voisinage et au-dessous de la température critique.
\end{abstract}

\begin{abstract}
The triangular lattice gas with repulsive nearest-neighbour interactions serves as a model for some adsorbed systems. We have applied renormalization-group methods to this model and obtained its phase diagram which is compared to that of $\mathrm{He}^{4}$ adsorbed on grafoil. Calculated values of the specific heat, with no adjustable parameters, agree remarkably well with experiment near the critical temperature and below.
\end{abstract}

1. Introduction. - The two-dimensional triangular lattice gas with nearest-neighbour repulsive interactions is of experimental interest because it is expected to provide a rather reasonable model for order-disorder transitions which occur in many physically adsorbed films. To be specific, let us consider the case of helium atoms adsorbed on exfoliated graphite, a system which has been studied extensively [1-4]. The carbon atoms of the substrate provide a triangular array of adsorption sites for the helium atoms. The latter are somewhat larger than the sites with the consequences that there is a repulsive interaction between atoms on neighbouring sites. At sufficiently high densities, then, one would expect the ground state of the system to be the ordered array shown in figure 1 . It follows that an order-disorder transition would be expected to be observed at a temperature of the order of magnitude of the repulsion. Such transitions have in fact been observed [1-4].

It is natural to consider these transitions within a lattice gas model and convenient to consider such a model in the magnetic language of an Ising system. The transcription is well known. At every site we associate a spin which is down if the site is occupied and $u p$ if the site is empty. The repulsion between atoms on adjacent sites then translates into an antiferromagnetic nearest-neighbour interaction.

The reduced hamiltonian of the system is

$$
-\beta \mathscr{H}_{\mathrm{o}}=K \sum_{i j} S_{i} S_{j}+H \sum_{i} S_{i}+\mathrm{NC}
$$

where $\beta$ is $\left(k_{\mathrm{B}} T\right)^{-1}$ and the first sum is over all nearest-neighbour pairs. $K=\beta J$ where $J$ is the usual exchange interaction. The reduced external magnetic field $H=\beta B$ is introduced so that the magnetization, which is equivalent to the density of the lattice gas, can be varied.

In order to obtain the ground state shown in figure 1 we must decompose the triangular net into three sublattices, denoted A, B, and C, which only contain spins which are second nearest-neighbours of one another. The expected configuration is then one in which the spins are up on two of the sublattices and down on the third. It should be noted that the magnetization of such a state is non-zero so that a magnetic field must be applied to obtain it. If there were no applied field there would be an equal number of up and down spins a circumstance which, in conjunction with the antiferromagnetic interaction and the triangular lattice prevents there from being any unique ground state at all. An 


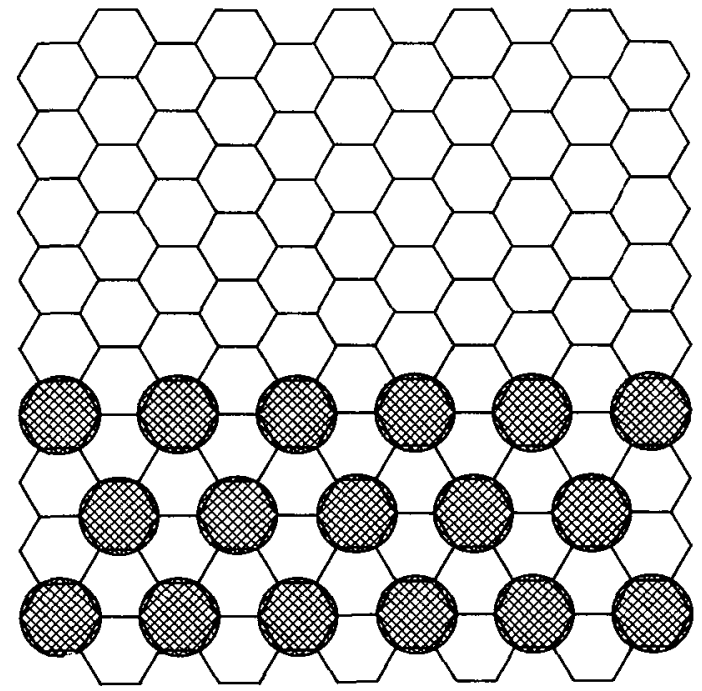

FIG. 1. - A representation of $\mathrm{He}^{4}$ atoms adsorbed on a graphite substrate. The ordered array shown in the lower half of the figure is expected over a range of coverages at low temperatures.

additional consequence is that there is no order-disorder transition at any finite temperature in the absence of an applied field. This initially surprising result is known from the exact solution [5] which may be constructed in zero magnetic field. In a field which is sufficiently strong, $|H / K|>6$, the ground state of the system is ferromagnetic so here, as for $H=0$, no transition is expected at finite temperature. However, a weak magnetic field, $6>|H / K|>0$, breaks the degeneracy while still favoring the antiferromagnetic ground state. Thus a transition from paramagnetic to antiferromagnetic states is expected at a field-dependent transition temperature $T_{c}(H / K)$. These considerations are borne out by Monte Carlo calculations [6]. Previous analytic approximations [7], however, which have been of the mean field type yield a finite transition temperature in zero field in disagreement with the exact result. Presumably this defect is due to the inability of such calculations to handle the large fluctuations associated with the ground state degeneracy.

We have applied to this problem the position-space renormalization group techniques of Niemeyer and van Leeuwen [8]. As emphasized by van Leeuwen [9] it is essential that the renormalization group operation, preserve the symmetry of the ordered ground state. To this end, we subdivide the lattice into $A, B, C$ sublattices and assign all spins to clusters as shown in figure 2 . Our approximation is to treat only three clusters, one from each sublattice, or nine spins in all. The cluster spin is assigned according to simple majority rule and periodic boundary conditions are employed. As is well known the renormalization group operation introduces interactions among the cell spins which

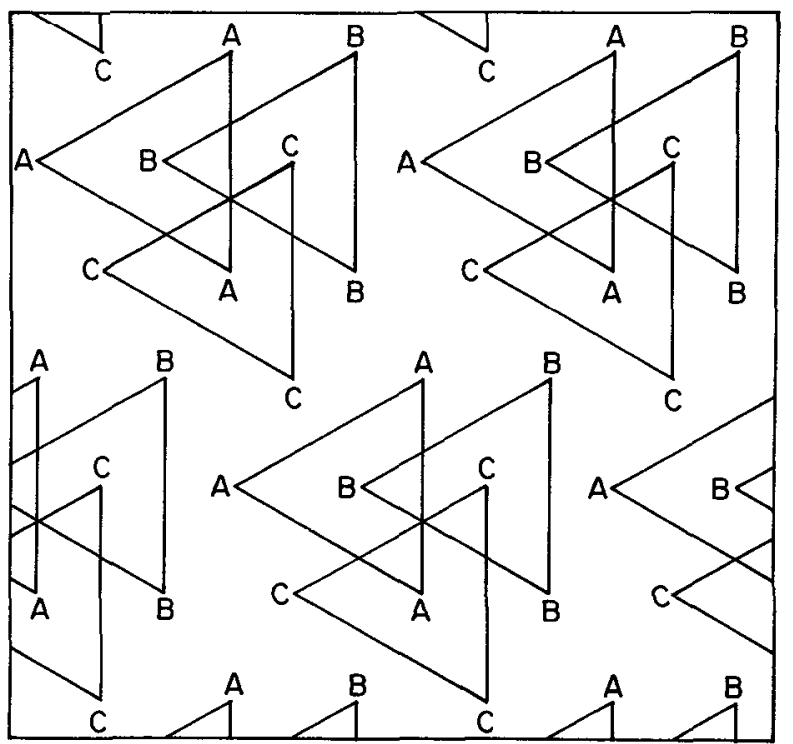

FIG. 2. - In the above the assignment of site spins to triangular cells is shown. Note that each cell only contains spins from one of the three sublattices.

are not present in the Hamiltonian of eq. (1). In our approximation only one additional interaction, $P$, is introduced which connects three spins which are all nearest-neighbours of one another. Thus our transformation preserves the form of the following Hamiltonian

$-\beta \mathscr{H}=K \sum_{i j} S_{i} S_{j}+H \sum_{i} S_{i}+P \sum_{i j k} S_{i} S_{j} S_{k}+\mathrm{NC}$

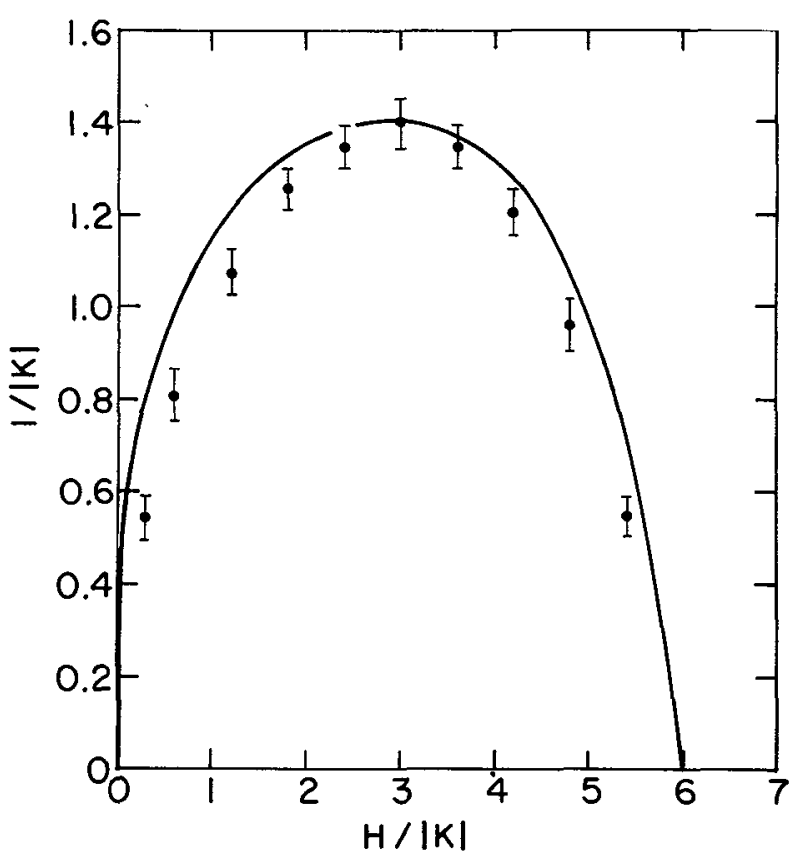

FiG. 3. - One half of the full phase diagram, which is symmetric about zero field, of the triangular Ising antiferromagnet is shown. The points and error bars are from the Monte Carlo calculation of ref. [6]. Temperature in units of $J, 1 / K$, is plotted versus magnetic field in the same units, $H / K$. 
which is symmetric under simultaneous reflection of $H$ and $P$. The details of the recursion relations produced by our approximation will be presented elsewhere.

In the space of the fields $K, H$, and $P$ the disordered paramagnetic phase is separated from the antiferromagnetically ordered state by a surface of phase transitions. It is the intersection of this surface with the surface of physical Hamiltonians $P=0$ which produces the line of transitions $T_{\mathrm{c}}(H / K)$ which we seek. Our result is shown in figure 3. Note that it produces the exact result $T_{c}(0)=0$. Also shown are the results of the Monte Carlo calculation. They are in rather good agreement.

In order to compare our result with adsorption

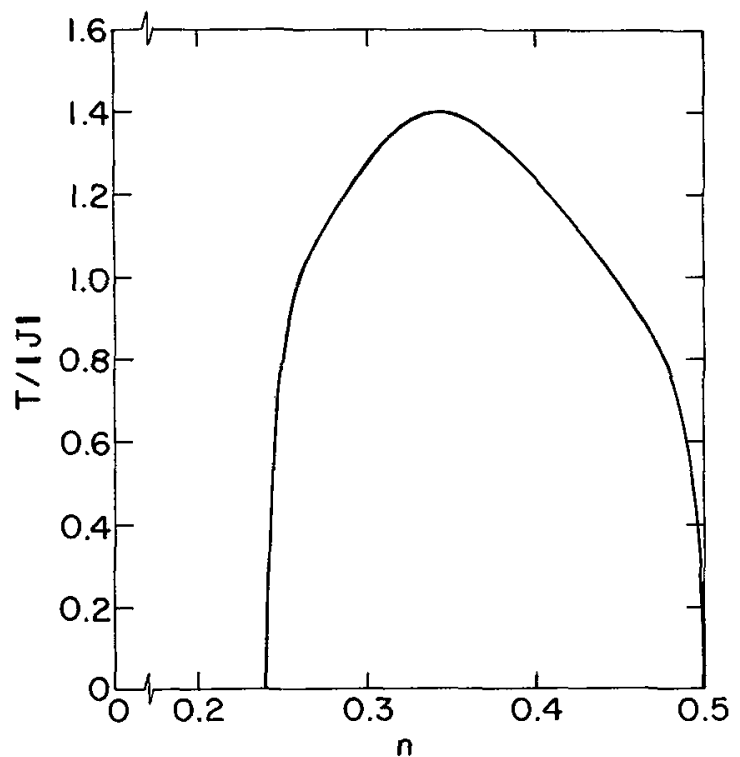

FIG. 4. - Phase diagram of the triangular lattice gas. As the full diagram is symmetric about a density of 0.5 only one half of it is shown. Temperature is plotted versus coverage, $n$.

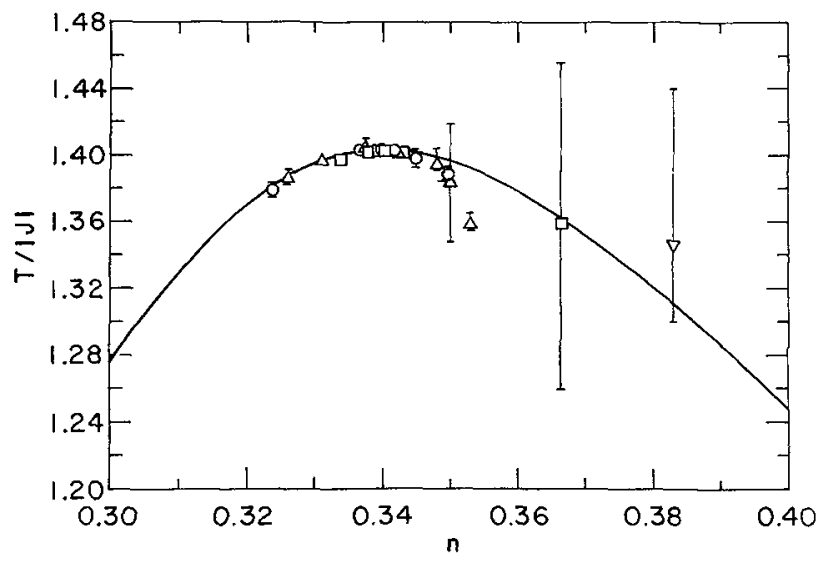

FIG. 5. - Comparison of calculated phase diagram with experimental results for $\mathrm{He}^{4}$ on Grafoil : circles $=$ cell $\mathrm{A}$, triangles $=$ cell $B$ both from ref. [1]; inverted triangle from ref. [2], squares from ref. [3]. experiments we calculate the magnetization $M(T, H)$ along the phase boundary thereby obtaining $T_{c}(M)$. We then make use of the relationship between $M$ and the coverage of the lattice gas $n, n=1 / 2[1+M]$, to obtain $T_{c}(n)$ which is shown in figure 4 . As the phase boundary is symmetric about $n=1 / 2$, only one half of it is shown. This phase diagram is compared in figure 5 to experimental results for $\mathrm{He}^{4}$ on Grafoil and on UCAR-ZYX an improved Grafoil-like substrate. We have determined the one adjustable parameter in our theory, $J$, by requiring that the maximum transition temperature be given correctly. This yields $|J| / k_{\mathrm{B}}=2.08 \mathrm{~K}$ which corresponds to a nearest-neighbour $\mathrm{He}^{4}-\mathrm{He}^{4}$ interaction of $8.32 \mathrm{~K}$, a reasonable value. It should be noted that our maximum transition temperature occurs at a density of 0.337 which is shifted due to entropy considerations from the energetically favored value of 0.333. As the experimental densities are not known to this accuracy we have placed the experimental density at which the maximum occurs at 0.337 . Comparison of our $T_{c}(n)$ with experiment at points other than the maximum provide a satisfactory test of our theory.

We have calculated the heat capacity per site at

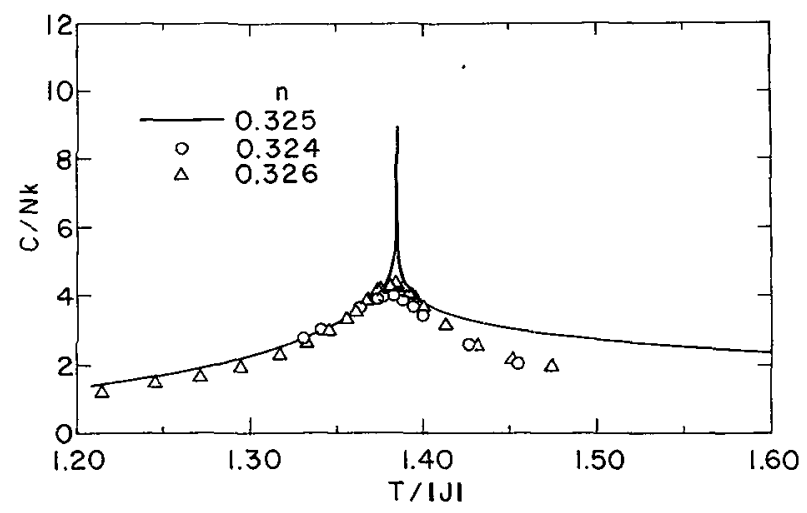

Fig. 6. - Heat capacity per particle at constant coverage. Data from ref. [1].

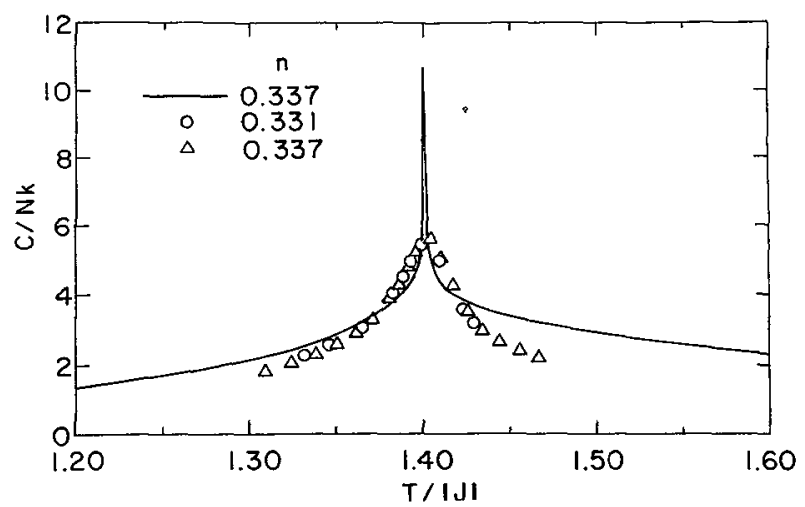

FIG. 7. - Heat capacity per particle at constant coverage. Data from ref. [1]. 
constant magnetization from which the constant-area heat capacity per particle of the lattice gas can be obtained. This heat capacity contains only the configurational contribution and not that from translational motion which we believe to be severly reduced in the ordered regime. Our results for several densities are shown in figures 5-9. It should be emphasized that once the value of $J$ and the calibration of the experimental densities have been determined from figure 5, there are no additional parameters to adjust. The agreement is excellent for $T<T_{c}$. The systematic discrepancies for $T>T_{\mathrm{c}}$ indicate that the assumptions of the lattice gas are breaking down at these temperatures.

It is interesting to observe from figure 9 that our results show, in agreement with experiment, that the singularity in the specific heat is more difficult to observe the further the density is from 0.337. Our calculations also show that for values of $n$ beyond that in figure 9 a secondary maximum appears in the specific heat. This maximum has nothing to do with the phase transition but may easily be incorrectly interpreted as its manifestation.

We turn now to the question of the nature of the singularity itself, that is, to the universality class to

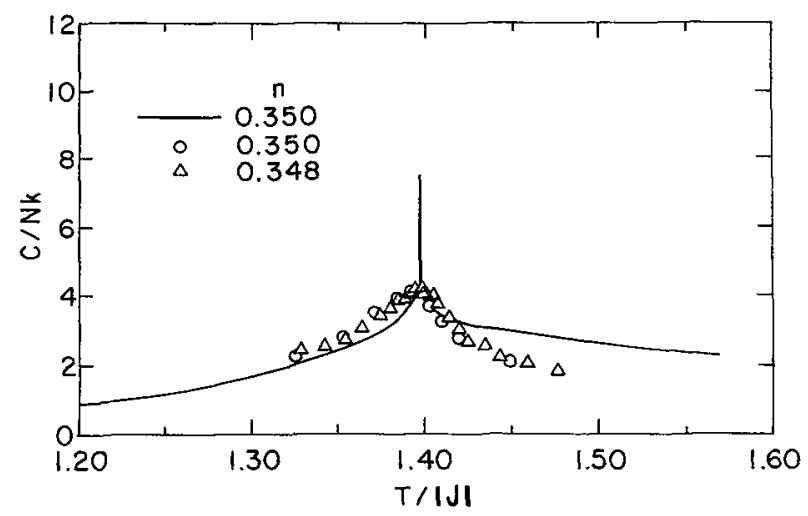

FIG. 8. - Heat capacity per particle at constant coverage. Data from ref. [1].

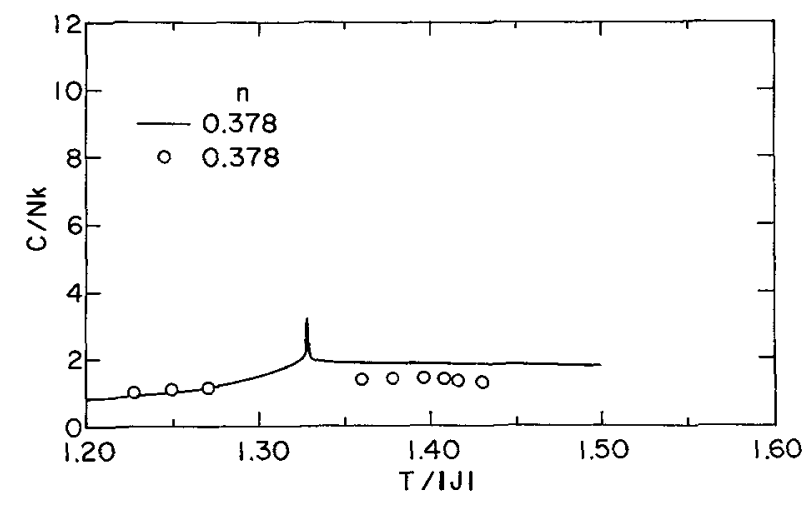

FIG. 9. - Heat capacity per particle at constant coverage. Data is from BRETZ, M., Ph. D. Thesis, University of Washington (1971), unpublished. which this transition belongs. It had long been assumed that because the system could be described by an Ising (classical spin 1/2) model that it would exhibit the logarithmic singularity of Onsager's solution of the ferromagnet [10]. In fact the early experimental data [1] supported this belief. It therefore came as a surprise when Alexander [11] showed that the transition should be in the class of the three-state Potts model [12]. The arguement is simple and may be phrased as follows. The ordered state of the system requires a two-component order parameter for its description. One component is needed to describe the difference between the occupation of the A and B sublattices and the other the difference in occupation of the $B$ and $C$ sublattices. These two components can be taken to be

$$
\begin{aligned}
& \psi_{1}=2 \sum n_{i}^{\mathrm{A}}-\sum n_{i}^{\mathrm{B}}-\sum n_{i}^{\mathrm{C}}, \\
& \psi_{2}=\sqrt{3}\left(\sum n_{i}^{\mathrm{B}}-\sum n_{i}^{\mathrm{C}}\right),
\end{aligned}
$$

where the $n_{i}$ 's are occupation numbers of the sites of the lattice gas or, in the language of the Ising model

$$
\begin{aligned}
\psi_{1} & =\sum S_{i}^{\mathrm{A}}-1 / 2 \sum S_{i}^{\mathrm{B}}-1 / 2 \sum S_{i}^{\mathrm{C}}, \\
\psi_{2} & =\sqrt{\frac{3}{2}}\left(\sum S_{i}^{\mathrm{B}}-\sum S_{i}^{\mathrm{C}}\right) .
\end{aligned}
$$

A Ginsburg-Landau-Wilson (GLW) Hamiltonian can now be constructed which is invariant under all the space group operations which leave the Hamiltonian of eq. (1) invariant. It is

$$
\mathscr{H}_{\mathrm{GLW}}=1 / 2 r \sum \psi_{i}^{2}+1 / 2 \sum\left(\nabla \psi_{i}\right)^{2}+w\left(\psi_{1}^{3}-3 \psi_{1} \psi_{2}^{2}\right)
$$

which is the GLW Hamiltonian of the three-state Potts model [13]. Bretz [3] has recently remeasured the specific heat exponent of the ordering transition and finds $\alpha=0.36$ which clearly indicates that the singularity is not logarithmic ( $\alpha=0$ ). The value of $\alpha$ for the three-state Potts model is not clear at this time for it has been calculated several times by different approximate methods with diverse results. A recent calculation of Burkhardt et al. [14], however, deserves mention. They employ the variational procedure of Kadanoff [15] which has given excellent exponent results for some transitions and obtain $\alpha=0.34$. The value of $\alpha$ which results from our calculation is obtained from the fixed point which governs the transition. We find $\alpha=-0.093$. Thus our specific heat results must differ from those of Bretz sufficiently close to the transition as shown in figure 10 .

The results presented above are those of a model calculation in which only nearest-neighbour repulsive interactions have been included. They have been applied to $\mathrm{He}^{4}$ on graphite because second 


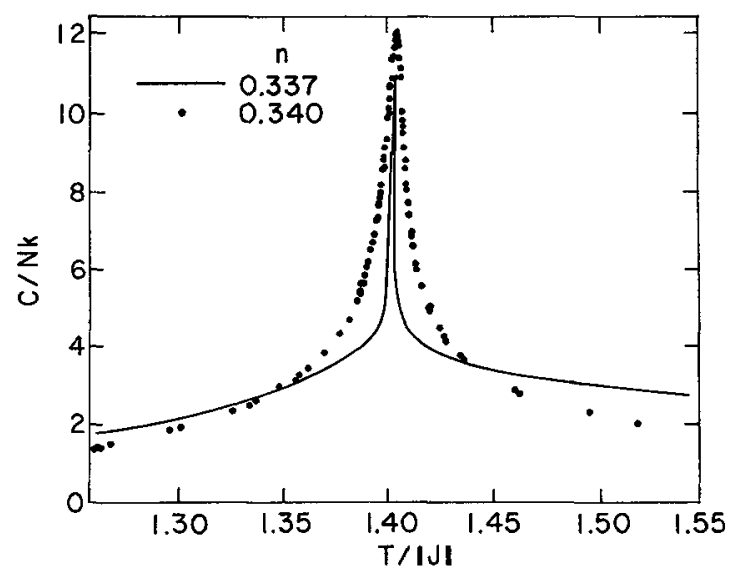

Frg. 10. - Heat capacity per particle at constant coverage. Data for $\mathrm{He}^{4}$ on the improved substrate UCAR-ZYX from ref. [3] at the density at which $T_{\mathrm{c}}$ is maximum. Theoretical curve is the same as in figure 7 .

nearest-neighbour interactions are expected to be weak. In other systems, these interactions may be rather strong so we briefly describe what their effect will be. At low temperatures there is a region of two-phase coexistence between an ordered phase of density near $1 / 3(2 / 3)$ and a disordered phase of density near zero (1). This coexistence region terminates in a tricritical point. In addition there is a region of coexistence between the ordered phase in which one sublattice is preferentially occupied and that in which two sublattices are so occupied. This coexistence region' culminates in a multicritical point [16]. Between these regions of coexistence for densities near $1 / 3(2 / 3)$ the transition remains second order with the same exponents as in the absence of the attractive interaction.

To recapitulate : we have applied position-space renormalization group methods to the triangular lattice gas with repulsive nearest-neighbour interactions. We have compared our results to experimental results obtained from $\mathrm{He}^{4}$ adsorbed on Grafoil and obtain good agreement for the variation of the transition temperature with density. Our results for the specific heat, calculated with no adjustable parameters, show remarkable agreement with experimental specific heats of the ordered phase except in the immediate neighbourhood of the transition.

\section{References}

[1] Bretz, M. et al., Phys. Rev. A 8 (1973) 1589.

[2] Hering, S.V. et al., J. Low Temp. Phys. 25 (1976) 793.

[3] BRETZ, M., Phys. Rev. Lett. 38 (1977) 501.

[4] DASH, J. G., Films on Solid Surfaces (Academic Press, New York), 1975.

[5] Houtappel, R. M. F., Physica 16 (1950) 425.

[6] Mercalf, B. D., Phys. Lett. 45A (1973) 1.

[7] Burley, D. M., Proc. Phys. Soc. 85 (1965) 1163 ; CampBELL, C. E. and Schick, M., Phys. Rev. A 5 (1972) 1919.

[8] Niemeyer, Th. and VAN Leeuwen, J. M. J., Phys. Rev. Lett. 31 (1973) 1411 ; Physica (Utr.) 71 (1974) 17.
[9] VAN Leeuwen, J. M. J., Phys. Rev. Lett. 16 (1975) 1056.

[10] Onsager, L., Phys. Rev. 65 (1944) 117.

[11] Alexander, S., Phys. Lett. 54A (1975) 353.

[12] Ports, R. B., Proc. Cambridge Philos. Soc. 48 (1952) 106.

[13] Straley, J. P. and Fisher, M. E., J. Phys. A 6 (1973) 1310.

[14] Burkhardt, T. W., et al,, J. Phys. A 9 (1976) L179.

[15] Kadanoff, L. P., Phys. Rev. Lett. 34 (1975) 1005.

[16] A Monte Carlo calculation of such a system which displays all these features is discussed by MIHURA, B. and Landau, D. P., Phys. Rev. Lett. 38 (1977) 977.

\section{DISCUSSION}

W. A. STEELE. - In real systems, the symmetries of these models will be broken by finite size effects and other imperfections. Is it possible to tell how these things will affect the critical exponents ?

M. SCHICK. - In general, no. Fisher and others have looked at finite size effects. Knowing the size of the sample, one could determine how close to $T_{\mathrm{c}}$ these effects would be manifest. If this were well within the critical region, one could simply exclude such data and use the remainder to determine the exponent. Thus finite size effects are not necessarily insurmountable.
J. P. McTague. - In lattice models, the disordered states are also pinned to lattice sites. Do the results reported here also apply to incommensurate disordered structures ?

M. SCHICK. - Yes. In the disordered state, the symmetry is that of the adsorption sites, regardless of whether the atoms are localised in sites or not. What we require only is that the ordered state be in registry with the substrate.

A. D. Novaco. - Is it necessary to use a lattice gas picture to do your classification calculation?

M. SCHICK. - No. 